\title{
DIAGNÓSTICO DEL ESTILO DE APRENDIZAJE PREDOMINANTE EN ESTUDIANTES DE CONTABILIDAD: RECONOCIMIENTO A LA DIVERSIDAD Y PUNTO DE PARTIDA PARA EL ENRIQUECIMIENTO PERSONAL
}

\author{
(Diagnostic testing on the prevalent learning style of accounting \\ students. Recognition of diversity and the starting point for personal
}

enrichment)

Pedro Osmany Laffita Azpiazú
pedroosmany@cug.co.cu

Eloy Guerrero Seide ${ }^{2}$ eloy@cug.co.cu

Fecha de recepción: II agosto de 2014 Fecha de aceptación: I5 septiembre de 2014

Se realizó un estudio descriptivo sobre los estilos de aprendizaje predominantes en los estudiantes del primer año del curso regular de la carrera Contabilidad y Finanzas de la Universidad de Guantánamo, Cuba, en el curso 2012-20 I3. Las variables consideradas fueron: Estilo de aprendizaje, edad y sexo. Se utilizó el cuestionario de Honey-Alonso (Alonso y Gallego, 2009) y como marco teórico la concepción de Kolb. La data fue procesada con el SPSS 20. Se realizaron análisis de diferencias significativas entre las puntuaciones medias poblacionales utilizando: La prueba $t$, para la variable estilos de aprendizaje, y las pruebas t y $U$ de Mann-Witney para el análisis de asociación entre estilo de aprendizaje y sexo. Se concluyó que en la población estudiada el estilo de aprendizaje predominante fue el reflexivo y el menos predominante el activo. No se halló asociación entre estilo de aprendizaje y sexo.

Palabras clave: Estilos de aprendizaje, Cuestionario Honey-Alonso, Modelo de Kolb, Prueba t, Prueba U de Mann-Witney.

\section{Abstract}

This study was carried out as a descriptive study about the predominant learning styles in first year's mainstream students who learn the Accounting Studies and Finance, at the University of Guantánamo, Cuba, during the academic 20I2-20I 3 course. The variables considered were: learning style, age and sex. The Honey-Alonso questionnaire and the Kolb's conception for a theoretical frame were used. The data was processed with the (SPSS) version 20. Datum was carried out through a rigid analysis and significant differences among the population's average punctuations were detected, using: the t-test, learning style variables; and the t-test and $U$ of Mann-Witney test in association between the learning style and sex. Concerning the studied population, it was concluded that the predominant learning style was reflexive and the less predominant was the active style. An association between the learning style and sex, was not found.

Key Words: Learning Styles, Questionnaire of Honey-Alonso, Kolb's Model, t-test, test U of MannWitney. 


\section{Introducción}

Dor lo general, los que investigan el tema aprendizaje concuerdan en que las personas aprenden de formas diferentes, sobre la base de su potencial, de sus conocimientos y de su experiencia. En esta perspectiva se considera que cada persona utiliza sus propios mecanismos para aprender, los que configuran en la persona que aprende una manera peculiar de procesar la información a la que se enfrenta y de transformarla en conocimiento. Para estas peculiares maneras de aprender se utiliza el término estilos de aprendizaje, concepto que fue acuñado por Herb Thelan en 1954 y que se ha ido desarrollando y creciendo en amplitud y complejidad en los años siguientes.

El tema de los estilos de aprendizaje tiene actualidad entre la comunidad de profesionales vinculados a los procesos educativos, tanto que se realiza desde el año 2004, y con una periodicidad de dos años, un congreso mundial sobre estilos de aprendizaje. Recientemente (durante los días 21, 22 y 23 de mayo de 2014) se celebró en la Pontificia Universidad Católica del Perú -PUCP, en Lima, el VI Congreso Mundial de Estilos de Aprendizaje y, próximamente (los días 7, 8 y 9 de octubre de 2015), se celebrará en Cartagena de Indias, Colombia, el III Congreso Iberoamericano de Estilos de Aprendizaje. Desde el año 2008 existe una revista sobre estilos de aprendizaje (http://learningstyles. uvu.edu); hay una página Web sobre el tema (http://www.estilosdeaprendizaje.es) y también una red sobre estilos de aprendizaje y educación a distancia (http://sites.google. com/site/estilosead/).

Actualmente se investiga sobre la relación de los estilos de aprendizaje con la edad, el sexo, las condiciones socioeconómicas, la escolaridad de los padres, las relaciones en- tre los estilos de quien enseña y de quien aprende; los estilos predominantes en diferentes cursos universitarios, y los estilos de aprendizaje y la educación a distancia, entre muchas otros temas de pesquisa que enriquecen cada día la teoría de los estilos de aprendizaje.

La vida ha mostrado y muestra los resultados de las prácticas de enseñanza que no toman en cuenta los estilos de aprendizaje que predominan en las personas que aprenden. Hoy se conoce que los problemas de disciplina, de rendimiento académico y de deserción escolar, entre muchos otros, se relacionan con el desarrollo de prácticas de enseñanza que no responden a los estilos de aprendizaje de los estudiantes.

La teoría de los estilos de aprendizaje constituye uno de los instrumentos favorecedores del reconocimiento de la diversidad dentro de las aulas, lo cual ha de constituir un punto de partida para el diseño de actividades de aprendizaje que fomenten el enriquecimiento personal de los sujetos.

Sobre los estilos se han desarrollado diferentes modelos teóricos y metodológicos, los que, desde diferentes perspectivas, ofrecen un marco conceptual y explicativo del comportamiento de la persona que aprende y del tipo de acción didáctica que puede resultar más eficaz en un momento dado del aprendizaje. Entre los modelos más conocidos y utilizados al respecto están:

I.Modelo de los cuadrantes cerebrales de Herrmann

2. Modelo de Felder y Silverman

3.Modelo de Kolb 
4.Modelo de Programación Neurolingüística de Bandler y Grinder

5.Modelo de los Hemisferios Cerebrales

6.Modelo de las Inteligencias Múltiples de Gardner

7.Modelo de los Enfoques de Aprendizaje

8.Enfoque Personológico en los Estilos de Aprendizaje

En este trabajo se asume el Modelo de Kolb sobre los estilos de aprendizaje, y se utiliza el Cuestionario Honey-Alonso-Cuestionario CHAEA- (Alonso y Gallego, 2009) para identificar el estilo de aprendizaje que predomina en los estudiantes de primer año (curso regular diurno) de la carrera Licenciatura en Contabilidad y Finanzas de la Universidad de Guantánamo, Cuba. Exponer los resultados de este estudio constituye el objetivo fundamental de este trabajo.

Las ideas de Kolb, desarrolladas por Honey-Mumford y Honey-Alonso, consideran que un aprendizaje óptimo requiere de cuatro fases: Experimentar, reflexionar, teorizar y actuar. Como generalidad, estas cuatro fases no se implementan por todos los sujetos en igual intensidad durante el proceso de aprendizaje. Incluso, en no pocos casos, ocurre que predomina una de ellas. De cierta manera, las fases predominantes vienen a caracterizar el modo de aprender de los individuos. Esta es la razón por la cual a las fases predominantes se les ha denominado estilos de aprendizaje de los sujetos. Siendo así, se pueden diferenciar, dentro del Modelo de Kolb, cuatro estilos de aprendizaje básicos: Pragmático, reflexivo, teórico y activo. En Gómez y Aduna (2004), se encuentran las principales características de los estudiantes que se identifican con estos estilos de aprendizaje; estas son:

\section{Alumnos activos}

Se involucran totalmente y sin prejuicios en las experiencias nuevas. Disfrutan el momento presente y se dejan llevar por los acontecimientos. Suelen ser entusiastas ante lo nuevo y tienden a actuar primero y pensar después en las consecuencias. Llenan sus días de actividades y tan pronto disminuye el encanto de una de ellas se lanzan a la siguiente. Les aburre ocuparse de planes a largo plazo y consolidar proyectos; les gusta trabajar rodeados de gente, pero siendo el centro de las actividades. La pregunta que quieren responder con el aprendizaje es icómo?

\section{Alumnos reflexivos}

Tienden a adoptar la postura de un observador que analiza sus experiencias desde muchas perspectivas distintas. Recogen datos y los analizan detalladamente antes de llegar a una conclusión. Para ellos lo más importante es esa recolección de datos y su análisis concienzudo, así que procuran posponer las conclusiones todo lo posible. Son precavidos y analizan todas las implicaciones de cualquier acción antes de ponerse en movimiento. En las reuniones observan y escuchan antes de hablar, procurando pasar desapercibidos. La pregunta que quieren responder con el aprendizaje es ipor qué?

\section{Alumnos teóricos}

Adaptan e integran las observaciones que realizan en teorías complejas y bien fundamentadas lógicamente. Piensan de forma secuencial y paso a paso, integrando hechos dispares en teorías coherentes. Les gusta analizar y sintetizar la información y su sistema de valores premia la lógica y la racionalidad. Se sienten incómodos con los juicios subjetivos, las técnicas de pensamiento late- 
ral y las actividades carentes de lógica clara. La pregunta que quieren responder con el aprendizaje es ¿qué?

\section{Alumnos pragmáticos}

Les gusta probar ideas, teorías y técnicas nuevas, y comprobar si funcionan en la práctica. Les gusta buscar ideas y ponerlas en práctica inmediatamente; les aburren e impacienta las largas discusiones sobre la misma idea de forma interminable. Son básicamente gente práctica, apegada a la realidad, a la que le gusta tomar decisiones y resolver problemas. Tales problemas son un desafío y siempre están buscando una mejor manera de hacer las cosas. La pregunta que quieren responder con el aprendizaje es ¿qué pasaría si...?

\section{Método}

Se realizó un estudio descriptivo de los estilos de aprendizaje en los estudiantes que recién ingresan a la carrera Licenciatura en Contabilidad y Finanzas de la Universidad de Guantánamo, Cuba, durante el curso escolar 2012-2013. Para el desarrollo de la pesquisa se trabajó con toda la población de estudiantes en la modalidad regular diurno, población compuesta por veintiocho (28) estudiantes que aceptaron participar de forma voluntaria en el estudio.

La variable en estudio (estilo de aprendizaje) fue medida utilizando el Cuestionario Honey-Alonso de estilos de aprendizaje (Ver Anexo), más conocido como Cuestionario CHAEA (Alonso y Gallego, 2009). Otras variables analizadas fueron la edad $y$ el sexo.
Los datos primarios fueron procesados con el SPSS, versión 20. El procesamiento consistió en la caracterización de la población atendiendo a la edad, el sexo y la variable principal en estudio; el análisis de las diferencias significativas entre las puntuaciones medias alcanzadas por la población de estudiantes en cada uno de los estilos de aprendizaje, utilizando la prueba $\mathrm{t}$ de students; y, por último, el análisis de las diferencias significativas entre las puntuaciones medias alcanzadas por los estudiantes de uno u otro sexo en cada estilo de aprendizaje, aplicando las pruebas $t$ de students y la no paramétrica $U$ de Mann-Witney.

\section{Resultados}

\section{Descripción de la población}

El Cuestionario CHAEA fue respondido por veintiocho (28) estudiantes, obteniéndose un coeficiente de confiabilidad Alfa de Cronbach de 0,843 para todo el instrumento.

Del total de estudiantes que respondieron el instrumento, quince (I5) estudiantes $(53,57 \%)$ fueron de sexo femenino y trece (13) estudiantes $(46,43 \%)$ de sexo masculino. Por otra parte; las edades en la población estudiada se ubican entre los valores 18 y 22 años; con una media de 19,46 años y desviación típica 0,92 años.

Análisis descriptivo y comparativo de los estilos de aprendizajes en la población de estudiantes

La Tabla I resume las cantidades de estudiantes que manifiestan el predominio de cada uno de los estilos de aprendizaje y de las posibles combinaciones de los mismos. 
Tabla I. Distribución de los estudiantes de acuerdo con los estilos de aprendizaje predominantes

\begin{tabular}{|l|c|c|}
\multicolumn{1}{|c|}{ ESTILOS } & FRECUENCIA ABSOLUTA & FRECUENCIA RELATIVA \\
\hline Activo & $\mathrm{I}$ & $3,57 \%$ \\
\hline Reflexivo & 12 & $42,86 \%$ \\
\hline Teórico & 5 & $17,86 \%$ \\
\hline Pragmático & $\mathrm{I}$ & $3,57 \%$ \\
\hline Activo, pragmático & $\mathrm{I}$ & $3,57 \%$ \\
\hline Activo, reflexivo & $\mathrm{I}$ & $3,57 \%$ \\
\hline Activo, teórico & $\mathrm{I}$ & $3,57 \%$ \\
\hline Reflexivo, pragmático & 4 & $14,26 \%$ \\
\hline Reflexivo, teórico & $\mathrm{I}$ & $3,57 \%$ \\
\hline Teórico, pragmático & $\mathrm{I}$ & $3,57 \%$ \\
\hline
\end{tabular}

Fuente: Autor, 2014.

En la tabla anterior se aprecia que existen nueve (9) estudiantes en los que predominan dos estilos de aprendizaje; en tanto que en diecinueve (19) de ellos predomina uno solo. También puede notarse que entre los estudiantes en los que predomina un único estilo de aprendizaje, la mayor frecuencia $(42,86 \%)$ corresponde al estilo reflexivo; mientras que la combinación de los estilos reflexivo y pragmático incorpora el mayor número $(14,26 \%)$ entre los estudiantes en los que predominan dos estilos de aprendizaje.

Partiendo de las respuestas a la encuesta aplicada se calculó la media y la desviación típica de las puntuaciones otorgadas a los estilos de aprendizaje de los estudiantes, las cuales se presentan en la Tabla 2.

Tabla 2. Media y desviación típica de las puntuaciones alcanzadas por los estudiantes pesquisados en cada estilo de aprendizaje

\begin{tabular}{|l|c|c|}
\hline \multicolumn{1}{|c|}{ ESTILO } & MEDIA & DESVIACIÓN TÍPICA \\
\hline Activo & 11,571 & 3,132 \\
\hline Reflexivo & 15,714 & 2,551 \\
\hline Teórico & 14,428 & 2,440 \\
\hline Pragmático & 13,500 & 2,715 \\
\hline
\end{tabular}

En la tabla anterior se observa que el estilo de aprendizaje reflexivo posee mayor media, resultado que concuerda con el alcanzando por otros investigadores de este tema en estudiantes de nivel superior, como por ejemplo Canalejas, Martínez y Pineda (2005), Montero, Sepúlveda y Contreras (abril de 20II) y Sepúlveda, López, y Torres, P. (abril de 20II).
El hecho de que el estilo reflexivo alcance mayor puntuación media induce a pensar en el predominio de este estilo de aprendizaje en el grupo de estudiantes pesquizados. No obstante; resulta necesario analizar la existencia $\circ$ no de diferencias significativas entre las puntuaciones medias alcanzadas en cada uno de los estilos de aprendizaje, para lo cual se utilizó la prueba $\mathrm{T}$ de students 
para dos poblaciones dependientes, previo análisis de la normalidad de las diferencias de medias correspondientes a los seis pares de combinaciones posibles entre los cuatro estilos de aprendizaje; es decir, activo y reflexivo, activo y teórico, activo y pragmático, reflexivo y teórico, reflexivo y pragmático y teórico y pragmático.

La prueba de Kolmogorov-Smirnov arrojó, con un $95 \%$ de confianza, el cumplimiento del supuesto de normalidad para las diferencias de medias correspondientes a los estilos activo y reflexivo (p: 0,846 ), activo y teórico ( $\mathrm{p}: 0,473)$, activo y pragmático (p: 0,534$)$, reflexivo y teórico (p: 0,777$)$, reflexivo y pragmático (p: 0,705) y teórico y pragmático (p: 0,592), razón por la cual se procedió a efectuar la prueba t de students.

Los resultados de la prueba $t$ de students indican rechazar la hipótesis de no existencia de diferencias significativas entre las puntuaciones medias alcanzadas entre los estilos activo y reflexivo ( $\mathrm{p}: 0,000$ ), activo y teórico (p: $0,00 \mathrm{I})$, activo y pragmático ( $\mathrm{p}$ : $0,002)$, reflexivo y teórico (p: 0,017) y, por último, reflexivo y pragmático ( $\mathrm{p}: 0,00 \mathrm{l})$. Respecto del análisis de las diferencias entre las medias alcanzadas en los estilos teórico y pragmático, la prueba $t$ de students indica aceptar la hipótesis de igualdad entre las medias contrastadas (p: 0,098). En todos los análisis efectuados se consideró un nivel de confianza del $95 \%$.

Análisis descriptivo y de asociación entre los estilos de aprendizaje y el sexo en la población de estudiantes pesquisados

La Tabla 3 resume el valor de la media aritmética y de la desviación típica correspondientes a las puntuaciones alcanzadas por los estudiantes de cada uno de los subgrupos determinados por las categorías (masculino y femenino) de la variable dicotómica sexo.

Tabla 3. Resultados de la prueba de normalidad para la variable cuantitativa en cada subgrupo determinado por la variable sexo

\begin{tabular}{|l|l|c|c|}
\hline \multicolumn{1}{|c|}{ ESTILO } & \multicolumn{1}{|c|}{ CATEGORÍAS } & MEDIA & $\begin{array}{c}\text { DESVIACIÓN } \\
\text { TÍPICA }\end{array}$ \\
\hline \multirow{2}{*}{ Activo } & Femenino & 12,067 & 2,865 \\
\cline { 2 - 4 } & Masculino & 11,000 & 3,440 \\
\hline \multirow{2}{*}{ Reflexivo } & Femenino & 16,333 & 2,637 \\
\cline { 2 - 4 } & Masculino & 15,000 & 2,345 \\
\hline \multirow{2}{*}{ Teórico } & Femenino & 14,933 & 2,086 \\
\cline { 2 - 4 } & Masculino & 13,846 & 2,764 \\
\hline Pragmático & Femenino & 13,467 & 3,044 \\
\cline { 2 - 4 } & Masculino & 13,539 & 2,402 \\
\hline
\end{tabular}

La prueba de Kolmogorov-Smirnov (ver Tabla 4) arrojó, con un $95 \%$ de confianza, el cumplimiento del supuesto de normalidad en los subgrupos correspondientes a los estilos teórico y pragmático; no así en los subgrupos correspondientes a los estilos activo y reflexivo. La tabla 4 resume los valores de vp (significación) obtenidos al efectuar esta prueba. 
Tabla 4. Resultados de la prueba de normalidad para la variable cuantitativa en cada subgrupo determinado por la variable sexo

\begin{tabular}{|l|l|c|}
\hline \multicolumn{1}{|c|}{ ESTILO } & \multicolumn{1}{|c|}{ CATEGORÍAS } & VALOR DE P \\
\hline \multirow{2}{*}{ Activo } & Femenino & 0,035 \\
\cline { 2 - 3 } & Masculino & 0,200 \\
\hline \multirow{2}{*}{ Reflexivo } & Femenino & 0,097 \\
\cline { 2 - 3 } & Masculino & 0,013 \\
\hline \multirow{2}{*}{ Teórico } & Femenino & 0,200 \\
\cline { 2 - 3 } & Masculino & 0,200 \\
\hline \multirow{2}{*}{ Pragmático } & Femenino & 0,200 \\
\cline { 2 - 3 } & Masculino & 0,200 \\
\hline
\end{tabular}

Fuente: Autor, 2014.

\section{Conclusiones}

La prueba de Levene indica aceptar la hipótesis de homogeneidad de las varianzas en los subgrupos determinados por la variable sexo y que corresponden a los estilos teórico (p: 0,256) y pragmático (p: 0,324). El cumplimiento de los supuestos de normalidad y homogeneidad de las varianzas permiten utilizar la prueba $t$ de students para el análisis de las diferencias entre las medias de los subgrupos determinados por la variable sexo en los estilos teórico y pragmático. Los resultados de esta prueba indican aceptar la hipótesis de igualdad de medias entre los subgrupos determinados por la variable sexo en los estilos teórico (p: 0,247) y pragmático (p: 0,946).

Por otra parte, el análisis de las diferencias entre las medias de los subgrupos determinados por la variable sexo en los estilos activo y reflexivo se efectuó por medio de la prueba U de Mann-Witney; la cual arrojó retener la hipótesis de que la distribución de los datos correspondientes a los estilos activo y reflexivo es la misma entre las categorías de la variable sexo (p: 0,618 para el estilo activo y $\mathrm{p:}$ 0, 156 para el estilo reflexivo). Todas las pruebas anteriores fueron realizadas al $95 \%$ de confianza.
Los resultados del análisis de las diferencias significativas entre las puntuaciones medias alcanzadas por la población de estudiantes en cada uno de los estilos de aprendizaje revelan que el perfil de los pesquisados manifiesta una tendencia hacia el predominio del estilo reflexivo, en contraposición a lo que sucede con el estilo de aprendizaje activo, pudiendo este ser considerado como aquel que menos predominio manifiesta entre la población objeto de estudio. Estos hallazgos deben tomarse en consideración a la hora de diseñar las actividades de aprendizaje con los estudiantes pesquisados.

Por otra parte, los resultados de la comparación de medias de cada estilo de aprendizaje entre los subgrupos determinados por las categorías de la variable sexo, permiten concluir que la media de los valores correspondientes a cada estilo de aprendizaje entre los subgrupos de estudiantes masculinos y femeninos no son estadísticamente diferentes; por tal razón, se afirma la no existencia de asociación entre los valores de las variables cuantitativas (valores de los estilos activo, reflexivo, teórico y pragmático) y los valores de la variable categórica sexo (masculino y femenino). 
I Licenciado en Educación con especialidad en Matemática, Universidad Pedagógica de Guantánamo, Cuba (1988); Máster en Didáctica de la Matemática, Universidad Pedagógica de Holguín, Cuba (1999); Doctor en Ciencias Pedagógicas (2008); Coordinador del Diplomado Estilos de Aprendizaje, Universidad de Guantánamo, Cuba.

2 Licenciado en Matemática, Universidad de Oriente (1979); Máster en Ciencias de la Educación Superior, Universidad de Oriente (1997); Doctorado en Ciencias Pedagógicas (200I); Docente, Universidad de Guantánamo, Cuba.

Referencias bibliográficas

Aguilera, E. y Ortiz, T. E. (abril de 20I0). La caracterización de perfiles de estilos de aprendizaje en la educación superior, una visión integradora. Revista estilos de aprendizaje, № 5 , Vol. 5.

Alonso, C. y Gallego, D. (2009) Cuestionario Honey-Alonso de Estilos de aprendizaje. Recuperado en Estilos de aprendizaje: http://www. estilosdeaprendizaje.es/menuprinc2.htm

Álvarez, D. y Domínguez, J. (200I). Estilos de aprendizaje en estudiantes de posgrado de una universidad particular. Revista Persona, Universidad de Lima, 4, I79-200.

Canalejas, M., Martínez, M. y Pineda, M. (2005). Estilos de aprendizaje en los estudiantes de Enfermería. Educación Médica. (8) 2, 83-90.

Chirino, N. y Padrón, E. (octubre de 20I I). La metacognición en los estilos de aprendizaje de estudiantes de postgrado durante la elaboración del trabajo de grado. Caso: La Universidad Rafael María Baralt -UNERMB. Revista Estilos de aprendizaje, (8) Vol. 8.

Coloma, C., Manrique, L., Revilla, D. y Tafur, R. (abril de 2008). Estudio descriptivo de los estilos de aprendizaje en docentes universitarios. Revista Estilos de aprendizaje, (I), I, I24-I4

Gómez, L. y Aduna, A. (2004). Manual de estilos de aprendizaje. Secretaría de Educación Pública. Subsecretaría de Educación Media Superior. Dirección General del Bachillerato. Dirección de Coordinación Académica.

Montero, E., Sepúlveda, M. y Contreras, E. (abril de 20I I): Estudio transversal de los estilos de aprendizaje y rendimiento académico de los alumnos de primer año de la carrera de Medicina Veterinaria. Revista Estilos de aprendizaje, (7) 7, I5I-I59

Morales, P. (2007). Estadística aplicada a las Ciencias Sociales: La fiabilidad de los test y escalas. Madrid: Universidad Pontificia Comillas.

Padilla, M., Akaela, M. et al. (abril de 20I3). Aproximación a los estilos de aprendizaje de diplomantes de la Universidad de las Ciencias Informáticas de la Habana, Cuba. Revista Estilos de aprendizaje, (I I) Vol. II.

Sepúlveda, M., López, M. y Torres, P. (abril de 20II). Diferencias de género en el rendimiento académico y en el perfil de estilos y de estrategias de aprendizaje en estudiantes de Química y Farmacia de la Universidad de Concepción. Revista Estilos de Aprendizaje, (7) 7, 13. 